\title{
Traducción, adaptación y validación al español del cuestionario de calidad de vida de 32 ítems (IBDQ-32) de la enfermedad inflamatoria intestinal
}

\author{
M. Masachs, F. Casellas y J. R. Malagelada \\ Unitat d'Atenció Crohn-Colitis. Hospital Universitari Vall d'Hebron. Ciberehd. Barcelona
}

\section{RESUMEN}

Introducción: la medida de la calidad de vida relacionada con la salud (CVRS) tiene una reconocida importancia en la evaluación, el manejo y el seguimiento de la enfermedad inflamatoria intestinal. El instrumento de medida más utilizado es la versión de 32 ítems del Inflammatory Bowel Disease Questionnaire (IBDQ32), que no está adaptado al español.

Objetivo: traducir la versión del IBDQ-32 al español y determinar su validez, fiabilidad y sensibilidad tanto en la colitis ulcerosa como en la enfermedad de Crohn.

Método: estudio prospectivo en dos fases: traducción y posterior validación del IBDQ-32 al español. La traducción se ha basado en la versión al español validada del IBDQ-36, y los ítems del IBDQ-32 no incluidos en el IBDQ-36 se tradujeron "de novo". Una vez terminada la traducción del IBDQ32, se aplicó un cuestionario específico de comprensión. Para determinar las propiedades psicométricas del IBDQ-32, un grupo de pacientes completó el IBDQ-36 ya validado al español y el IBDQ-32.

Resultados: se han incluido 84 pacientes (53 con enfermedad de Crohn y 31 con colitis ulcerosa). La mediana de la puntuación global de ambos cuestionarios en los 84 pacientes no fue diferente ( 6,1 vs. $6,2, p=n s)$ y su correlación de Spearman fue muy significativa $(r=0,97, p<0,001)$. La capacidad de discriminar entre pacientes con brote y remisión también fue equivalente en ambos cuestionarios (6,4 vs. 6,4 con $r=0,96$ en remisión y 3,8 vs. 3,9 con $r=0,95$ en actividad, $p<0,001$ remisión vs actividad). Estos resultados se reprodujeron en el análisis para EC y CU independientemente $(6,4$ vs. 6,4 y 6,7 vs. 6,6 respectivamente, $p=n s)$.

Conclusión: la versión en castellano de 32 ítems del IBDQ es válida y discriminativa para ser aplicada en pacientes con enfermedad de Crohn o colitis ulcerosa.

Palabras clave: Enfermedad de Crohn. Colitis ulcerosa. Epidemiología. Calidad de vida. Cuestionario de calidad de vida. Validación.

\section{Recibido: 17-04-07.}

Aceptado: 11-05-07.

Financiado en parte por 2005SGR00398 de la Generalitat de Catalunya Departament d'Universitats, Recerca i Societat de l'Informació. Instituto de Salud Carlos III (Ciberehd, España).

Correspondencia: Francesc Casellas. Servei de Digestiu. Hospital Universitari Vall d'Hebrón. Ciberehd. Passeig de la Vall d'Hebrón, 119. 08035 Barcelona.e-mail: fcasellas@ vhebron.net

\begin{abstract}
Introduction: the measurement of health-related quality of life (HRQoL) has an established relevance in the assessment, management, and follow-up of inflammatory bowel disease. The most commonly used measuring instrument is the 32-item version of Inflammatory Bowel Disease Questionnaire (IBDQ-32), which has never been adapted to Spanish.

Objective: to translate IBDQ-32 into Spanish, and to establish its validity, reliability, and sensitivity both in ulcerative colitis and Crohn's disease.

Method: a prospective study in two phases -translation into Spanish and subsequent validation of IBDQ-32. Translation was based on the validated Spanish version of IBDQ-36, and IBDQ32 items not included in IBDQ-36 were translated from scratch. Once the IBDQ32 translation was completed a comprehensionspecific questionnaire was administered. To establish IBDQ-32 psychometric properties a group of patients completed both the validated Spanish version of IBDQ-36 and IBDQ-32.

Results: eighty-four patients (53 with Crohn's disease and 31 with ulcerative colitis) were included. Median overall scores in both questionnaires for all 84 patients did not differ (6.1 vs. 6.2, $\mathrm{p}=\mathrm{ns})$, and Spearman's correlation was highly significant $(\mathrm{r}=$ $0.97, p<0.001$ ). The ability to discriminate between patients in flare-up or remission was also equivalent for both questionnaires (6.4 vs. 6.4 with $r=0.96$ in remission and 3.8 vs. 3.9 with $r=$ 0.95 active, $p<0.001$ for remission vs. activity). These results were obtained in both the analyses for $\mathrm{CD}$ and UC independently (6.4 vs. 6.4 and 6.7 vs. 6.6, respectively; $p=n s$ ).
\end{abstract}

Conclusion: the Spanish version of IBDQ-32 is valid and discriminating for patients with Crohn's disease or ulcerative colitis.

Key words: Crohn's disease. Ulcerative colitis. Epidemiology. Quality of life. Quality of life questionnaire. Validation.

Masachs M, Casellas F, Malagelada JR. Traducción, adaptación y validación al español del cuestionario de calidad de vida de 32 ítems (IBDQ-32) de la enfermedad inflamatoria intestinal. Rev Esp Enferm Dig 2007; 99: 511-519. 


\section{INTRODUCCIÓN}

El objetivo de las intervenciones médicas es tratar la enfermedad y restablecer la salud de las personas. En este sentido, el tratamiento médico de las enfermedades crónicas está dirigido básicamente a mejorar los síntomas, reducir la morbilidad o descender la mortalidad. Recientemente se ha reconocido que conseguir una mejor percepción de la calidad de vida es muy beneficioso y un punto clave en el tratamiento de las enfermedades crónicas. Por ello, el campo de la calidad de vida relacionada con la salud (CVRS) ha ganado protagonismo en la medicina y especialmente en la gastroenterología. Este interés es reflejado en el factor que el sujeto es regularmente citado en la literatura médica (1).

Basados en la definición de la Organización Mundial de la Salud (2), la percepción de la salud tiene que ser considerada en diferentes dimensiones de la vida. Síntomas orgánicos, o sus efectos, son uno de los dominios básicos de la CVRS. Hay no obstante, muchas otras dimensiones como son las funcionales, sociales, psicológicas, económicas, espirituales, laborales, etc. La valoración de la influencia de salud en conjunto o en dimensiones específicas es valorada usualmente utilizando cuestionarios.

Existen cuestionarios genéricos de aplicación general y cuestionarios específicos. Estos últimos son específicos para una enfermedad, grupo de población o cierta función, condición o problema. Los instrumentos específicos de una enfermedad tienen las ventajas de su mayor sensibilidad y de estar relacionados a áreas analizadas de rutina por los clínicos. Existen instrumentos específicos desarrollados para evaluar la CVRS de diferentes enfermedades, entre ellas la enfermedad inflamatoria intestinal.

La enfermedad de Crohn (EC) y la colitis ulcerosa (CU) son enfermedades inmumoinflamatorias que ocupan un lugar importante para los pacientes, sus familiares y la sociedad $(3,4)$. Basados en la definición de salud para la Organización Mundial de Salud, EC y CU son enfermedades crónicas que influencian las dimensiones físicas, psicológicas, familiares y sociales de la vida. Las implicaciones físicas y psicológicas se manifiestan como discomfort emocional o físico, cambios sexuales, pérdida de la independencia, cambios en la percepción de vida, miedo a perder el control y las consecuencias de ello, etc. Las implicaciones familiares se manifestaran, entre otras, por pérdida del rol familiar, cambios en las responsabilidades de la familia o distanciamiento de la familia mientras se está en el hospital. Las implicaciones sociales se manifestaran por incidentes como cambios de lugar en el trabajo, aislamiento social o restricciones en los salarios o en las actividades de ocio. Las implicaciones en la CVRS son acentuadas por el requerimiento de tratamientos prolongados y sus efectos adversos, la necesidad de resecciones quirúrgicas con ostomías potenciales, visitas periódicas físicas y eventuales hospitalizaciones. En este sentido, la valoración de la CVRS juega un papel importante no sólo en la evaluación de las consecuencias de la enfermedad inflamatoria intestinal sobre la CVRS sino también en la evaluación del efecto del tratamiento.
La enfermedad inflamatoria intestinal deteriora permanentemente la CVRS del paciente, este impacto es más pronunciado durante los brotes y mejora en los períodos de remisión, no obstante, no alcanza los niveles de CVRS normales que presenta la población (5). La CVRS está influenciada por determinados factores dependientes de la enfermedad como la actividad, la necesidad de hospitalización o el número de brotes anuales. Otros factores independientes reconocidos como el sexo femenino o el nivel educacional también existen (6). En cualquier caso, el factor más importante en la CVRS en la EII son los brotes de la enfermedad, afectando la CVRS del sujeto tanto en la percepción cualitativa como cuantitativa $(7,8)$. No obstante, experiencias pasadas con la EII no influencian significativamente con la percepción de salud (9).

Varios instrumentos han sido utilizados para valorar la CVRS en la EII. El cuestionario más utilizado es la versión de 32 ítems del Inflammatory Bowel Disease Questionnaire (IBDQ-32) (10) y la versión ampliada de 36 ítem del Inflammatory Bowel Disease Questionnaire (IBDQ-36) (11). La Unitat d'Atenció Crohn-Colitis (UACC) del Hospital Universitari Vall de Hebron ha diseñado y validado un cuestionario corto basado en el cuestionario IBDQ-36 que incluye 9 ítems, con unas adecuadas propiedades psicométricas (12) y correlación con la actividad endoscópica en los pacientes con CU (13).

El IBDQ ha sido diseñado y validado para la lengua y cultura inglesa. Para su adaptación transcultural al español se tienen que establecer las propiedades psicométricas del cuestionario traducido en el contexto de la nueva cultura y lengua $(14,15)$. Atendiendo dichas consideraciones metodológicas, sólo el IBDQ-36 ha sido traducido y validado al español (16). No obstante, otros países han escogido la versión adaptada del IBDQ-32. La ausencia de una versión española del IBDQ-32 debidamente traducida, adaptada a la lengua española y validada, limita a los centros españoles a participar en estudios internacionales e impide la comparación con los resultados de CVRS obtenidos en otros países de nuestro entorno. Por ello, el objetivo del presente estudio ha sido traducir al castellano el IBDQ-32, validar su comprensibilidad en dicha lengua y las propiedades psicométricas del cuestionario.

\section{MATERIAL Y MÉTODO}

\section{Pacientes}

Se ha propuesto la participación a un grupo de pacientes atendidos en la UACC. Un investigador explicó los objetivos del estudio y los pacientes dieron su consentimiento oral para participar en el estudio. Los pacientes que no aceptaron participar, por no saber leer o no completar los cuestionarios, fueron excluidos del estudio.

Los pacientes incluidos presentaron tanto EC como CU. El diagnóstico se estableció según los criterios de clínica, endoscopia, radiología e histología compatible (17). Se recogieron los datos demográficos (edad, sexo, 
tabaco, situación laboral, nivel educativo) y clínicos básicos de la enfermedad inflamatoria intestinal.

Para determinar la actividad clínica de la EC se utilizó el índice de Harvey-Bradshaw (18) y para la CU el índice de Rachmilewitz (19). Ambos índices están basados en variables clínicas como el número de deposiciones, la presencia de dolor abdominal, el estado general, complicaciones, fiebre o manifestaciones extraintestinales de la enfermedad. Se consideró la enfermedad activa cuando el índice de Harvey-Bradshaw fue mayor de 2 o cuando el índice de Rachmilewitz fue superior de 6.

\section{Procedimiento}

Para la traducción del cuestionario original se ha partido de la versión ya traducida y adaptada al español IBDQ-36, con lo que para la mayoría de preguntas no se ha precisado una nueva traducción. Para las preguntas del IBDQ-32 no incluidas en el IBDQ-36 español se ha procedido a su traducción por expertos bilingües. Para garantizar la adecuada comprensión de los nuevos ítems traducidos, una vez terminada la traducción completa del IBDQ-32, se realizó un cuestionario específico de comprensión (¿ha tenido problemas para entender la pregunta?, ¿qué significado tiene la pregunta para usted?, ¿piensa que la pregunta es importante en su situación actual?, ¿cómo plantearía usted la pregunta?, ¿cree que hay una relación lógica entre la pregunta y la respuesta?), que se administró a un número reducido de pacientes. En el Anexo se muestra el cuestionario IBDQ-32 con todos los ítems incluidos y sus respuestas.

Para determinar las propiedades psicométricas de la versión IBDQ-32 española, se ha utilizado como referencia el único cuestionario específico para la medida de la calidad de vida en la enfermedad inflamatoria intestinal validado al castellano, el IBDQ-36 (16). Cada paciente completó dos cuestionarios de CVRS: el validado previamente IBDQ-36 y el nuevo cuestionario IBDQ-32. El cuestionario escogido como test de oro o de referencia ha sido la versión española del IBDQ-36, que es el único cuestionario específico traducido y validado al español (16). Contiene 36 ítems distribuidos en 5 dimensiones (síntomas digestivos, síntomas sistémicos, afectación emocional, social y afectación funcional). Las respuestas de cada ítem están divididas en 7 puntos, dónde el número 7 es el nivel más alto y el número 1 el más bajo. El instrumento produce una puntuación global para la totalidad de los ítems y 5 puntuaciones para cada una de las dimensiones. El cuestionario IBDQ-32 contiene 32 ítems distribuidos en 4 dimensiones (síntomas digestivos, síntomas sistémicos, afectación emocional y social). El IBDQ-32 no contiene la dimensión de la afectación funcional de la enfermedad. Las respuestas de cada ítem se expresan en una escala de 7 puntos, donde 7 corresponde a la mejor percepción de CVRS y 1 a la peor. El instrumento produce una puntuación global para la totalidad de los ítems y 4 puntuaciones para cada una de las dimensiones.

\section{Análisis estadístico}

Las variables se describen como medianas y percentiles 25-75. Las diferencias entre medianas se establecieron utilizando el test de Mann-Whitney o Kruskal-Wallis. Las correlaciones se calcularon utilizando el test de correlación de Spearman's. El nivel aceptado de significación estadística fue del $5 \%(\mathrm{p}<0,05)$.

\section{RESULTADOS}

Se han incluido un total de 84 pacientes, 53 pacientes con EC y 31 con CU. Las características de la población se recogen de forma detallada en la tabla I. La mediana de la edad de la población estudiada es de 38 años (3743), similar para la CU 45 (43-53) y la EC 32 (32-39). La distribución por sexos muestra un discreto predominio masculino con 46 varones y 38 mujeres. Se observa también un predominio de fumadores en la EC. En cuanto a la localización de la enfermedad había un predominio de pancolitis seguido de proctocolitis y colitis izquierda en los pacientes con colitis ulcerosa. En los pacientes con enfermedad de Crohn la localización más frecuente fue la ileocólica e íleon terminal. En el momento de realizar el cuestionario la mayor parte de pacientes se encontraba en remisión clínica ( 73 frente a $27 \%$ que estaba en fase activa).

En cuanto al resultado global de los cuestionarios, la mediana de la puntuación global del IBDQ-32 en los 84 pacientes no fue diferente de la del IBDQ-36 (6,1 vs. 6,2, $\mathrm{p}=\mathrm{ns}$ ) (Tabla II) y su correlación de Spearman fue muy

Tabla I. Características de los pacientes incluidos en el estudio. Los resultados se describen como medianas (percentiles 25-75) o valores absolutos (\%)

\begin{tabular}{|c|c|c|c|}
\hline Variables & Total & Colitis ulcerosa & E. de Crohn \\
\hline $\begin{array}{l}\text { Número de pacientes } \\
\text { Edad } \\
\text { Género (varón/mujer) }\end{array}$ & $\begin{array}{c}84 \\
38 \\
46 / 38\end{array}$ & $\begin{array}{c}31 \\
45(43-53) \\
17 / 14\end{array}$ & $\begin{array}{c}53 \\
32(32-39) \\
21 / 32\end{array}$ \\
\hline \multicolumn{4}{|l|}{ Hábito tabáquico } \\
\hline $\begin{array}{l}\text { Fumador } \\
\text { Ex fumador } \\
\text { No fumador } \\
\text { Localización }\end{array}$ & $\begin{array}{l}34(40 \%) \\
14(17 \%) \\
36(43 \%)\end{array}$ & $\begin{array}{c}7(23 \%) \\
5(16 \%) \\
19(61 \%) \\
\text { Proctitis (E1) } 10 \\
\text { Colitis izq. (E2) } 9 \\
\text { Pancolitis (E3) } 12\end{array}$ & $\begin{array}{c}27(51 \%) \\
9(17 \%) \\
17(32 \%) \\
\text { Illeon (L1) } 18 \\
\text { Colon (L2) } 11 \\
\text { Ileocolon (L3) } 21 \\
\text { G-I alto (L4) } 2\end{array}$ \\
\hline $\begin{array}{l}\text { Actividad (\%) } \\
\text { Remisión } \\
\text { Brote }\end{array}$ & $\begin{array}{l}68(81 \%) \\
16(19 \%)\end{array}$ & $\begin{array}{c}27(87 \%) \\
4(13 \%)\end{array}$ & $\begin{array}{l}41(77 \%) \\
12(23 \%)\end{array}$ \\
\hline $\begin{array}{l}\text { Indice de actividad clín } \\
\text { Remisión } \\
\text { Brote }\end{array}$ & & $\begin{array}{c}1(0,6-1,9) \\
6(5,4-7)\end{array}$ & $\begin{array}{l}1(0,6-1,1) \\
6(4,8-7,9)\end{array}$ \\
\hline Meses de evolución & $108(93-124)$ & $126(89-145)$ & $108(85-122)$ \\
\hline $\begin{array}{l}\text { Indice actividad } \\
\text { (brotes/año) }\end{array}$ & $0,49(0,5-0,77)$ & $1(0,8-2,3)$ & $1(1,36-2,8)$ \\
\hline
\end{tabular}


Tabla II. Resultados obtenidos en los cuestionarios IBDQ-36 e IBDQ-32 para el conjunto total de los 84 pacientes, descritos como medianas y percentiles

\begin{tabular}{lcc}
\hline & IBDQ-36 & IBDQ-32 \\
\hline Global & $6,2(5,3-5,9)$ & $6,1(5,3-5,9)$ \\
Digestivo & $6,1(5,5-6)$ & $6,2(5,6-6,1)$ \\
Sistémico & $5,6(5,1-5,6)$ & $5,6(4,8-5,5)$ \\
Emocional & $6,2(5,2-5,9)$ & $6,1(5,2-5,8)$ \\
Funcional & $6,4(5,3-6)$ & \\
Social & $6,5(5,6-6,1)$ & $6,8(5,5-6,3)$ \\
\hline
\end{tabular}

Tabla III. Correlaciones obtenidas entre las puntaciones de los cuestionarios IBDQ-36 e IBDQ-32 para el conjunto de los 84 pacientes

\begin{tabular}{lcc}
\hline & $r$ Spearman & $p$ \\
\hline Global & 0,97 & $<0,001$ \\
Digestivo & 0,97 & $<0,001$ \\
Sistémico & 0,93 & $<0,001$ \\
Emocional & 0,84 & $<0,001$ \\
Funcional & & $<0,001$ \\
Social & 0,85 & $<$ \\
\hline
\end{tabular}

Tabla IV. Resultados obtenidos en los cuestionarios IBDQ-36 e IBDQ-32 en los pacientes con enfermedad de Crohn, descritos como medianas y percentiles

\begin{tabular}{lcc}
\hline & IBDQ-36 & IBDQ-32 \\
\hline Global & $6,4(5,5-6,1)$ & $6,4(5,5-6,1)$ \\
Digestivo & $6,6(5,6-6,2)$ & $6,6(5,6-6,2)$ \\
Sistémico & $6,1(5,3-5,9)$ & $6(5,1-5,8)$ \\
Emocional & $6,6(5,4-6,1)$ & $6,3(5,4-6)$ \\
Funcional & $6,6(5,5-6,1)$ & \\
Social & $6,6(5,6-6,3)$ & $6,8(5,6-6,4)$ \\
\hline
\end{tabular}

Tabla V. Resultados obtenidos en los cuestionarios IBDQ-36 e IBDQ-32 en los pacientes con colitis ulcerosa, descritos como medianas y percentiles

\begin{tabular}{lcc}
\hline & IBDQ-36 & IBDQ-32 \\
\hline Global & $6,7(5,6-6,2)$ & $6,6(5,6-6,2)$ \\
Digestivo & $6,8(5,6-6,2)$ & $6,8(5,6-6,3)$ \\
Sistémico & $6,6(5,5-6,1)$ & $6,6(5,4-6,1)$ \\
Emocional & $6,7(5,4-6,2)$ & $6,6(5,5-6,1)$ \\
Funcional & $6,8(5,5-6,2)$ & \\
Social & $6,7(5,6-6,3)$ & $7(5,6-6,3)$ \\
\hline
\end{tabular}

Tabla VI. Resultados obtenidos en los cuestionarios IBDQ-36 e IBDQ-32 en los pacientes con efermedad de Crohn y colitis ulcerosa, divididos en remisión y en brote, descritos como medianas y percentiles

\begin{tabular}{ccccc}
\hline & \multicolumn{2}{c}{ Enfermedad de Crohn } & \multicolumn{2}{c}{ Colitis ulcerosa } \\
& Remisión & Brote & Remisión & Brote \\
\hline IBDQ-32 & $6,4(5,8-6,3)$ & $4,1(3,4-4,8)$ & $6,4(5,5-6,3)$ & $3,6(2,8-4,3)$ \\
& & $p<0,001$ & & $p<0,001$ \\
IBDQ-36 & $6,4(5,7-6,3)$ & $4(3,3-4,9)$ & $6,4(5,6-6,4)$ & $3,6(2,5-4,4)$ \\
& & $p<0,001$ & & $p<0,001$ \\
\hline
\end{tabular}

significativa ( $\mathrm{r}=0,97, \mathrm{p}<0,001$ ) (Tabla III), lo que sugiere la adecuada equivalencia entre ambos cuestionarios. Los resultados por dimensiones (síntomas digestivos, síntomas sistémicos, afectación emocional y afectación social) tampoco difirieron entre el IBDQ-32 y el IBDQ-36 (Tabla II). La capacidad de discriminar entre pacientes con brote y remisión también fue similar para ambos cuestionarios (6,4 vs. 6,4 con $r=0,96$ en remisión y 3,8 vs. 3,9 con $\mathrm{r}=0,95$ en actividad, $\mathrm{p}<0,001$ remisión $\mathrm{y}$ actividad) lo que demuestra que el nuevo IBDQ-32 tiene una adecuada capacidad discriminativa.

En cuanto a los resultados del análisis para EC y CU independientemente, la mediana de la puntuación global del IBDQ-36 no fue diferente de la del IBDQ-32 (6,4 vs. 6,4 y 6,7 vs. 6,6 para la EC y la CU respectivamente, $\mathrm{p}=$ ns) (Tablas IV y V) y la correlación de Spearman entre ambos cuestionarios fue muy significativa ( $\mathrm{r}=0,97 \mathrm{y} \mathrm{p}<$ 0,0001 para la EC y $r=0,98$ y p $<0,0001$ para la CU). Los resultados por dimensiones (síntomas digestivos, síntomas sistémicos, afectación emocional y afectación social) tampoco difirieron entre el IBDQ-36 y el IBDQ-32 al analizarlo independientemente para EC y CU (Tablas IV y V). La capacidad de discriminar entre pacientes con EC en brote o remisión fue similar para ambos cuestionarios. En los pacientes con EC en remisión, la puntuación de los cuestionarios fue de 6,4 vs . 6,4 para el IBDQ-36 y IBDQ-32 respectivamente, con una correlación entre ambos cuestionarios de $r=0,94$ ( $\mathrm{p}<0,001)$, y para los pacientes con EC en brote, de 4 vs. 4,1 para el IBDQ-36 y IBDQ-32 respectivamente, con una $\mathrm{r}=0,99(\mathrm{p}<0,001)$ entre ambos cuestionarios.

Estos resultados se reprodujeron en la CU (Tablas VI y VII). En los pacientes con CU en remisión, la puntuación de los cuestionarios fue de 6,4 vs. 6,4 para el IBDQ-36 y IBDQ-32 respectivamente, con una correlación entre ambos cuestionarios de $\mathrm{r}=0,98(\mathrm{p}<0,001) \mathrm{y}$, para los pacientes con $\mathrm{CU}$ en brote, de 3,6 vs. 3,6 para el IBDQ-36 y IBDQ-32 respectivamente, con una $r=0,40(p>0,05)$ entre ambos cuestionarios. La existencia de las mencionadas diferencias entre las puntuaciones del IBDQ-32 en función del grado de actividad, dan validez al constructo del nuevo cuestionario tanto en los pacientes con EC como con CU. En las figuras 1 y 2 se describe la correla-

Tabla VII. Correlaciones obtenidas entre los cuestionarios IBDQ-36 e IBDQ-32 en los pacientes con enfermedad de Crohn y colitis ulcerosa según el grado de actividad de la enfermedad

\begin{tabular}{lcc}
\hline & r Spearman & $p$ \\
\hline $\begin{array}{l}\text { Enfermedad de Crohn } \\
\text { Global }\end{array}$ & 0,99 & \\
Remisión & 0,94 & 0,001 \\
Actividad & 0,99 & 0,001 \\
Colitis ulcerosa & & 0,001 \\
Global & 0,98 & \\
Remisión & 0,98 & 0,001 \\
Actividad & 0,40 & 0,001 \\
\end{tabular}




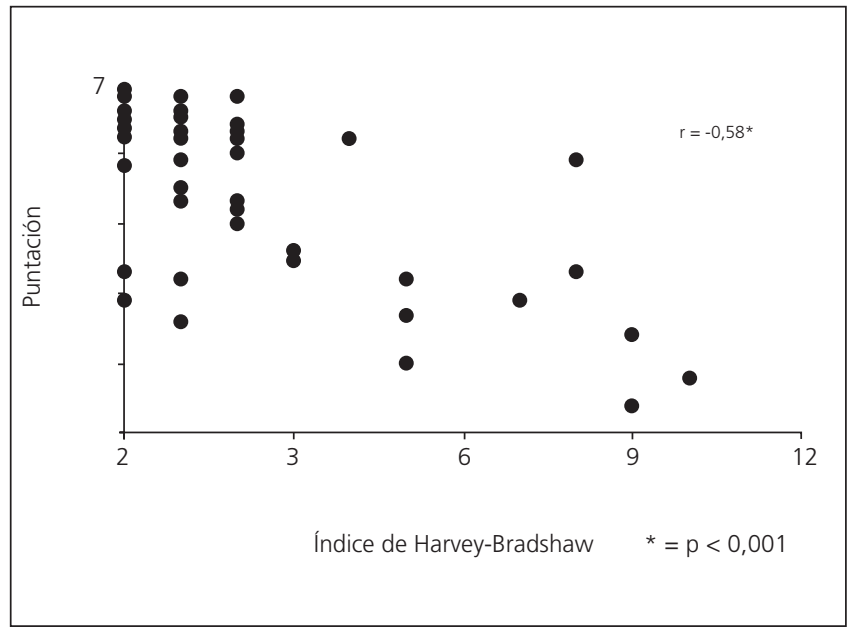

Fig. 1. Correlación del índice de actividad clínica con la puntuación global del cuestionario IBDQ-32 en los pacientes con enfermedad de Crohn.

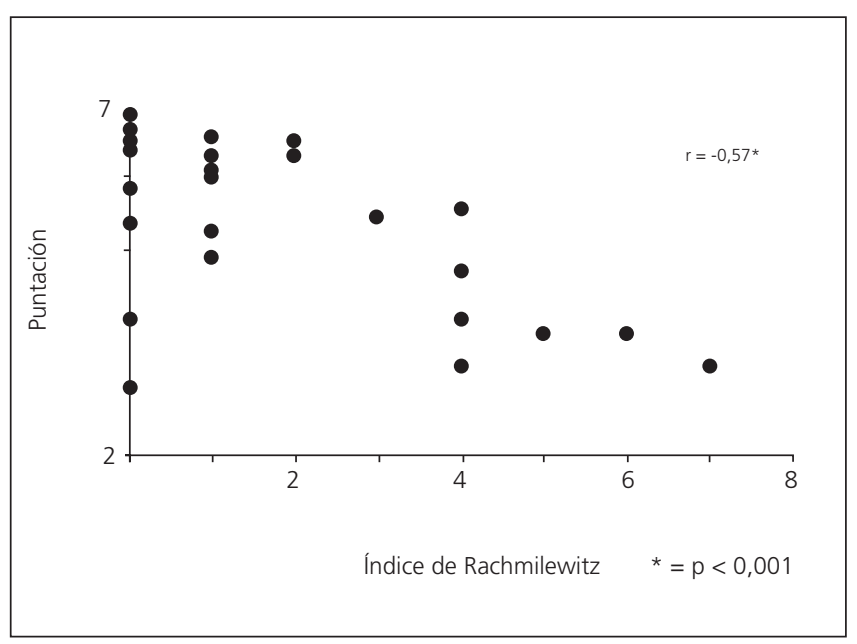

Fig. 2. Correlación del índice de actividad clínica con la puntuación global del cuestionario IBDQ-32 en los pacientes con colitis ulcerosa.

ción entre el índice de actividad clínica y la puntuación del IBDQ-32. Se observa que existe una correlación significativa, cambiando los resultados de la puntuación global del cuestionario en paralelo con el índice clínico de actividad.

\section{DISCUSIÓN}

La EII comporta una afectación de la salud, que se manifiesta en forma de cambios en las actitudes y conductas a nivel físico, emocional y social. El estudio de la CVRS en la EII permite no sólo un mejor conocimiento de la enfermedad y de las repercusiones que conlleva para el paciente, sino que además tiene importantes repercusiones aparte de las puramente clínicas. Se ha observado que la afectación de la CVRS en la EII

\section{Anexo. Cuestionario IBDQ-32 traducido, adaptado y validado al español}

MARQUE CON UN CÍRCULO LA RESPUESTA QUE CORRESPONDE MÁS EXACTAMENTE CON SU SITUACIÓN DURANTE LAS ÚLTIMAS DOS SEMANAS

1. ¿Con qué frecuencia ha ido de vientre durante las últimas dos semanas?

1. Más frecuentemente que nunca

2. Extremada frecuencia

3. Con mucha frecuencia

4. Moderado aumento de la frecuencia de defecación

5. Ligero aumento de la frecuencia de defecación

6. Aumento mínimo de la frecuencia de defecación

7. Normal, sin ningún aumento de la frecuencia de defecación

2. ¿Con qué frecuencia le ha causado problemas la sensación de fatiga o de cansancio y agotamiento durante las últimas dos semanas?

$\square$ 1. Siempre

2. Casi siempre

$\square$ 3. Bastantes veces

$\square$ 4. A veces

5. Pocas veces

6. Casi nunca

$\square$ 7. Nunca

3. ¿Con qué frecuencia se ha sentido frustrado, impaciente o inquieto a causa de su problema intestinal durante las últimas dos semanas?

$\square$ 1. Siempre

2. Casi siempre

3. Bastantes veces

4. A veces

5. Pocas veces

6. Casi nunca

$\square$ 7. Nunca

4. ¿Con qué frecuencia se ha visto incapacitado para ir a estudiar o al trabajo a causa de su problema intestinal durante las últimas dos semanas?

$\square$ 1. Siempre

2. Casi siempre

3. Bastantes veces

4. A veces

5. Pocas veces

6. Casi nunca

7. Nunca

5. ¿Durante cuánto tiempo en las últimas dos semanas ha tenido diarrea?

$\square$ 1. Siempre

2. Casi siempre

3. Bastantes veces

4. A veces

5. Pocas veces

6. Casi nunca

$\square$ 7. Nunca

6. ¿Cuánta energía ha tenido durante las últimas dos semanas?

1. Ninguna energía

2. Muy poca energía

3. Poca energía

4. Cierta energía

5. Bastante energía

6. Mucha energía

7. Rebosante de energía 
7. ¿Con qué frecuencia ha estado preocupado ante la posibilidad de tener que operarse por su problema intestinal durante las últimas dos semanas?

\section{Siempre \\ 2. Casi siempre \\ 3. Bastantes veces \\ $\square$ 4. A veces \\ 5. Pocas veces \\ 6. Casi nunca \\ - 7. Nunca}

8. ¿Con qué frecuencia ha tenido que aplazar o anular una cita o compromiso social a causa de su problema intestinal durante las últimas dos semanas?

\section{$\square$ 1. Siempre \\ 2. Casi siempre \\ 3. Bastantes veces \\ - 4. A veces \\ 5. Pocas veces \\ $\square$ 6. Casi nunca \\ 7. Nunca}

9. ¿Con qué frecuencia ha tenido retortijones durante las últimas dos semanas?

a 1. Siempre

2. Casi siempre

3. Bastantes veces

4. A veces

- 5. Pocas veces

- 6. Casi nunca

7. Nunca

10. ¿Con qué frecuencia ha tenido malestar general durante las últimas dos semanas?

$\square$ 1. Siempre

2. Casi siempre

3. Bastantes veces

4. A veces

5. Pocas veces

$\square$ 6. Casi nunca

7. Nunca

11. ¿Con que frecuencia ha estado preocupado por temor a no encontrar un lavabo cerca durante las últimas dos semanas?

$\square$ 1. Siempre

2. Casi siempre

3. Bastantes veces

4. A veces

5. Pocas veces

$\square$ 6. Casi nunca

7. Nunca

12. ¿Qué dificultad ha tenido, a causa de su problema intestinal, en las actividades de ocio o deportes que le hubiera gustado hacer durante las últimas dos semanas?

$\square$ 1. Muchísima dificultad; imposible hacer actividades

2. Mucha dificultad

3. Bastante dificultad

$\square$ 4. Algo de dificultad

5. Un poco de dificultad

6. Apenas ninguna dificultad

7. Ninguna dificultad; mi problema intestinal no ha limitado mis actividades de ocio ni deportivas
13. ¿Con qué frecuencia ha tenido dolor abdominal durante las últimas dos semanas?
$\square$ 1. Siempre
2. Casi siempre
3. Bastantes veces
$\square$ 4. A veces
5. Pocas veces
6. Casi nunca
7. Nunca

14. ¿Con qué frecuencia ha tenido problemas porque se ha despertado por la noche durante las últimas dos semanas?
1. Siempre
2. Casi siempre
3. Bastantes veces
4. A veces
5. Pocas veces
$\square$ 6. Casi nunca
7. Nunca

15. ¿Con qué frecuencia se ha sentido deprimido o desanimado a causa de su problema intestinal durante las últimas dos semanas?
1. Siempre
2. Casi siempre
3. Bastantes veces
4. A veces
5. Pocas veces
$\square$ 6. Casi nunca
7. Nunca

16. ¿Con qué frecuencia durante las últimas dos semanas ha tenido que dejar de asistir a actos sociales porque no había un lavabo cerca?

$\square$
$\square$ 1. Siempre
2. Casi siempre
3. Bastantes veces
$\square$ 4. A veces
$\square$ 5. Pocas veces
$\square$ 6. Casi nunca
$\square$ 7. Nunca

17. En general, ¿hasta qué punto ha sido un problema tener gases durante las últimas dos semanas?
$\square$ 1. Un gran problema
2. Un problema importante
3. Bastante problemático
$\square$ 4. Algo problemático
5. Muy poco problemático
6. Casi ningún problema
7. Ningún problema

18. En general, ¿hasta qué punto ha sido un problema durante las últimas dos semanas el mantener o llegar al peso que a $\mathrm{Vd}$. le gustaría?

$\square$ 1. Un gran problema

2. Un problema importante

- 3. Bastante problemático

4. Algo problemático

- 5. Muy poco problemático

6. Casi ningún problema

7. Ningún problema 
19. Muchos pacientes con un problema intestinal tienen frecuentes preocupaciones y angustias a causa de su enfermedad. En general, ¿con qué frecuencia durante las últimas dos semanas se ha sentido preocupado o angustiado por llegar a tener cáncer, o por pensar que nunca más volvería a encontrarse bien, o por tener una recaída?

\section{Siempre \\ 2. Casi siempre \\ 3. Bastantes veces \\ $\square$ 4. A veces \\ 5. Pocas veces \\ 6. Casi nunca \\ $\square$ 7. Nunca}

20. ¿Con qué frecuencia durante las últimas dos semanas ha tenido una sensación de hinchazón abdominal?

$\square$ 1. Siempre

2. Casi siempre

3. Bastantes veces

4. A veces

5. Pocas veces

6. Casi nunca

$\square$ 7. Nunca

21. ¿Con qué frecuencia durante las últimas dos semanas se ha sentido relajado y libre de tensión?

\section{$\square$ 1. Nunca \\ $\square$ 2. Casi nunca \\ 3. Pocas veces \\ 4. A veces \\ 5. Bastantes veces \\ 6. Casi siempre \\ 7. Siempre}

22. ¿Cuántas veces durante las últimas dos semanas ha tenido problemas de sangrar al ir de vientre?
$\square$ 1. Siempre
2. Casi siempre
3. Bastantes veces
4. A veces
5. Pocas veces
6. Casi nunca
7. Nunca

23. ¿Con qué frecuencia durante las últimas dos semanas se ha sentido avergonzado en público por olores desagradables o ruidos causados por su problema intestinal?
$\square$ 1. Siempre
2. Casi siempre
3. Bastantes veces
4. A veces
5. Pocas veces
6. Casi nunca
7. Nunca

24. ¿Con qué frecuencia durante las últimas dos semanas ha tenido ganas de ir al lavabo sin realmente hacer de vientre?

$\square$ 1. Siempre

2. Casi siempre

3. Bastantes veces

$\square$ 4. A veces

5. Pocas veces

6. Casi nunca

$\square$ 7. Nunca
25. ¿Con qué frecuencia se ha sentido deprimido, Iloroso o desanimado a causa de su problema intestinal durante las últimas dos semanas?
1. Siempre
2. Casi siempre
3. Bastantes veces
4. A veces
5. Pocas veces
6. Casi nunca
$\square$ 7. Nunca

26. ¿Con qué frecuencia durante las últimas dos semanas ha manchado accidentalmente su ropa interior?
$\square$ 1. Siempre
2. Casi siempre
3. Bastantes veces
4. A veces
5. Pocas veces
- 6. Casi nunca
$\square$ 7. Nunca

27. ¿Con qué frecuencia durante las últimas dos semanas se ha sentido enfadado a causa de su problema intestinal?
$\square$ 1. Siempre
2. Casi siempre
3. Bastantes veces
4. A veces
5. Pocas veces
6. Casi nunca
- 7. Nunca

28. En general, durante las últimas dos semanas, ¿hasta qué punto su problema intestinal ha sido un problema para sus relaciones sexuales?

$\square$ 1. Un gran problema

2. Un problema importante

3. Bastante problemático

$\square$ 4. Algo problemático

5. Muy poco problemático

6. Casi ningún problema

7. Ningún problema

29. ¿Con qué frecuencia ha tenido náuseas o ganas de vomitar durante las últimas dos semanas?
1. Siempre
2. Casi siempre
3. Bastantes veces
4. A veces
5. Pocas veces
b. Casi nunca
7. Nunca

30. ¿Con qué frecuencia se ha sentido de mal humor durante las últimas dos semanas?

$\square$ 1. Siempre

2. Casi siempre

a 3. Bastantes veces

4. A veces

5. Pocas veces

6. Casi nunca

7. Nunca 
31. ¿Con qué frecuencia durante las últimas dos semanas se ha sentido incomprendido por los demás?

$\square$ 1. Siempre
$\square$ 2. Casi siempre
3. Bastantes veces
$\square$ 4. A veces
$\square$ 5. Pocas veces
$\square$ 6. Casi nunca
7. Nunca

32. ¿Hasta qué punto ha estado satisfecho, contento o feliz con su vida personal durante las últimas dos semanas?

$\square$ 1. Muy insatisfecho, infeliz

2. Bastante insatisfecho, infeliz

3. Algo insatisfecho, descontento

4. Algo satisfecho, contento

5. Bastante satisfecho, contento

6. Muy satisfecho, feliz

7. Extremadamente satisfecho, no podría ser más feliz fueron diferentes de las del IBDQ-36 lo que demuestra una buena equivalencia con el "estándar de oro" previamente disponible. Durante la realización del presente manuscrito se ha publicado un trabajo de validación del IBDQ-32 que confirman las adecuadas propiedades psicométricas del cuestionario pero sin hacer referencia a la validación lingüística y que, además usa como referencia para la valoración de las propiedades cuestionarios no específicos para la enfermedad inflamatoria intestinal sino cuestionarios de evaluación psicológica (23).

En conclusión, la versión en castellano de 32 ítems del IBDQ es válida y discriminativa para ser la medida de la CVRS de los pacientes con enfermedad de Crohn o colitis ulcerosa.

\section{BIBLIOGRAFÍA}

tiene valor predictivo en cuanto al uso de recursos sanitarios (20), es un buen marcador del efecto de las intervenciones terapéuticas sobre la enfermedad (21) y se incluye de forma rutinaria en los análisis farmacoeconómicos (22). Todo ello sugiere que el conocimiento del impacto de la EII sobre la CVRS de los enfermos es de gran utilidad por sus implicaciones médicas, sociales y de distribución de los recursos sanitarios.

La mayoría de los cuestionarios de medida de la CVRS en la EII han sido elaborados y validados para su aplicación específica en la población angloparlante. La simple traducción literal de un cuestionario a otro idioma no garantiza su fiabilidad, sino que siempre deben determinarse la comprensibilidad de los ítems a la lengua traducida y las propiedades psicométricas de todo cuestionario traducido antes de su aplicación. En este sentido, el IBDQ ha sido traducido y debidamente validado a diferentes idiomas, incluido el castellano. En castellano se dispone de la versión de 36 ítems del IBDQ y de la versión reducida de 9 ítems debidamente validadas. Pero el instrumento más utilizado actualmente a nivel internacional es la versión de 32 ítems del IBDQ (IBDQ-32) que, al no estar debidamente traducida y validada al español, ha motivado la realización del presente estudio. Para simplificar el proceso de traducción, los ítems que se comparten en las versiones IBDQ-32 y IBDQ-36 se han aprovechado la versión previamente validada al español del IBDQ-32. Las preguntas del IBDQ-32 no incluidas en el IBDQ36 se han traducido por personal angloparlante y posteriormente se ha determinado la comprensibilidad de dichos ítems.

A continuación se han determinado las propiedades psicométricas del IBDQ-32. El nuevo cuestionario ha demostrado poseer la capacidad de discriminar entre pacientes con brote y remisión, tanto en la EC como en la CU. Por otro lado, las puntuaciones del IBDQ-32 no
1. Casellas F, López Vivancos J, Vergara M, Malagelada JR. Impact of inflammatory bowel disease on health-related quality of life. Dig Diseases 1999; 17: 208-18.

2. Casellas F, Fontanet G, Borruel N, Malagelada JR. Opinión de los pacientes con enfermedad inflamatoria intestinal sobre la atención sanitaria recibida. Rev Esp Enferm Dig 2004; 96: 174-84.

3. Cohen RD. The quality of life in patients with Crohn's disease. Aliment Pharmacol Ther 2002; 16: 1603-9.

4. Vergara M, Casellas F, Badia X, Malagelada JR. Assessing the quality of life of household members of patients with inflammatory bowel disease: Development and validation of a specific questionnaire. Am J Gastroenterol 2002; 97: 1429-37.

5. Casellas F, López Vivancos J, Badia X, Vilaseca J, Malagelada JR. Impact of surgery for Crohn's disease on health-related quality of life. Am J Gastroenterol 2000; 95: 177-82.

6. Casellas F, López Vivancos J, Casado A, Malagelada JR. Factors affecting health-related quality of life of patients with inflammatory bowel disease. Quality Life Research 2002; 11: 775-81.

7. Casellas F, López Vivancos J, Badia X, Vilaseca J, Malagelada JR. Influence of inflammatory bowel disease on different dimensions of quality of life. Eur J Gastroenterol Hepatol 2001; 13: 567-72.

8. Casellas F, Arenas JI, Baudet JS, Fabregas S, García N, Gelabert J, et al. Impairment of health-related quality of life in patients with inflammatory bowel disease: A Spanish multicenter study. Inflamm Bowel Dis 2005; 11: 488-96.

9. Casellas F, López Vivancos J, Malagelada JR. Experiencia previa y calidad de vida en pacientes con enfermedad inflamatoria intestinal durante los brotes de actividad. Rev Esp Enferm Dig 2003; 95: 471-5.

10. Guyatt G, Mitchell A, Irvine EJ, et al. A new measure of health stalogy 1989; 96: 804-10.

11. Love JR, Irvine EJ, Fedorak RN. Quality of life in inflammatory bowel disease. J Clin Invest 1992; 14: 15-9.

12. Alcalá MJ, Casellas F, Fontanet G, Prieto L, Malagelada JR. Shortened questionnaire on quality of life for inflammatory bowel disease. Inflamm Bowel Dis 2004; 10: 383-91.

13. Casellas F, Alcalá MJ, Prieto L, Armengol JR, Malagelada JR. Assessment of the influence of disease activity on the quality of life of patients with inflammatory bowel disease using a short questionnaire. Am J Gastroenterol 2004; 99: 457-61.

14. Guillemin F, Bombardier C, Beaton D. Cross-cultural adaptation of health-related quality of life instruments: Literature review and proposed guidelines. J Clin Epidemiol 1994; 46: 1417-32.

15. Herdman M, Fox Rushby J, Badia X. A model of equivalence in the cultural adaptation of HRQol instruments: The universalist approach. Qual Life Research 19987: 323-35. tus for clinical trials in inflammatory bowel disease. Gastroentero- 
16. López Vivancos J, Casellas F, Badía X, Vilaseca J, Malagelada JR. Validation of the Spanish version of the Inflammatory Bowel Disease Questionnaire in ulcerative colitis and Crohn's disease. Digestion 1999: 60: 274-80.

17. Hodgson HJF, Bhatti M. Assessment of disease activity in ulcerative colitis and Crohn's disease activity. Inflamm Bowel Dis 1995; 1: 117-43.

18. Harvey RF, Bradshaw JM. A simple index of Crohn's disease activity. Lancet 1980; 1: 514.

19. Rachmilewitz D. Coated mesalazine (5-aminosalicylic acid) versus sulphasalazine in the treatment of active ulcerative colitis: A randomized trial. Br Med J 1989; 298: 82-6.
20. De Boer AG, Sprangers MA, Bartelsman J, de Haes JC. Predictors of health care utilization in patients with inflammatory bowel disease: A longitudinal study. Eur J Gastroenterol Hepatol 1998; 10: 783-9.

21. Irvine EJ. Quality of life issues in patients with inflammatory bowel disease. Am J Gastroenterol 1997;92 (Supl. 12): S18-S24.

22. Feagan BG. Review article: Economic issues in Crohn's diseaseassessing the effets of new tretments on health-related quality of life. Aliment Pharmacol Ther 1999; 13 (Supl. 4): 29-37.

23. Vidal A, Gómez Gil E, Sans M, Portella MJ, Salamero M, Piqué $\mathrm{JM}$, et al. Propiedades psicométricas del cuestionario de la enfermedad inflamatoria intestinal: Una versión española. Gastroenterol Hepatol 2007; 30: 212-8. 